\title{
Diferentes manifestações imaginológicas da displasia cemento-óssea: relato de dois
}

\section{casos clínicos}

\author{
Different imaginological manifestations of cementobone dysplasia: report of two clinical cases \\ Diferentes manifestaciones imaginológicas de displasia cementobónica: informe de dos casos
}

clínicos

Recebido: 04/11/2021 | Revisado: 12/11/2021 | Aceito: 19/11/2021 | Publicado: 20/11/2021

\author{
Jéssica Lorrayne Nunes Silva \\ ORCID: https://orcid.org/0000-0003-3588-1533 \\ Faculdade Independente do Nordeste, Brasil \\ E-mail: nunesjessicalsn@gmail.com \\ Lívia Saldanha Gomes Lima \\ ORCID: https://orcid.org/0000-0002-3342-0832 \\ Faculdade Independente do Nordeste, Brasil \\ E-mail: liviasaldanha@hotmail.com \\ Yasmin Correia Coelho \\ ORCID: https://orcid.org/0000-0001-5799-6492 \\ Faculdade Independente do Nordeste, Brasil \\ E-mail: yasmincoelho1992@gmail.com \\ Brenda Tigre Rocha \\ ORCID: https://orcid.org/0000-0002-8234-1882 \\ Faculdade Independente do Nordeste, Brasil \\ E-mail: brenda.tigre.rocha@gmail.com \\ Juliana Borba Santos \\ ORCID: https://orcid.org/0000-0001-6516-3458 \\ Faculdade Independente do Nordeste, Brasil \\ E-mail: julianaborba@fainor.com.br \\ Luísa Soares Santino Correia \\ ORCID: https://orcid.org/0000-0002-5704-1044 \\ Faculdade Independente do Nordeste, Brasil \\ E-mail: luisasantino@fainor.com.br \\ Lara Correia Pereira \\ ORCID: https://orcid.org/0000-0002-5529-9260 \\ Faculdade Independente do Nordeste, Brasil \\ E-mail: lcp.larapereira@gmail.com
}

\begin{abstract}
Resumo
Objetivo: Relatar dois casos clínicos, distintos, de displasia cemento óssea periapical, com manifestações radiográficas diferentes e mesma conduta odontológica para a sua resolução. Metodologia: Foram descritos dois casos clínicos, de pacientes do sexo feminino, melanodermas, entre a terceira e quarta décadas de vida, encaminhadas para uma clínica escola do Sudoeste da Bahia para avaliação endodôntica. Após os exames radiográficos, na primeira paciente, foi observada área radiolúcida em região periapical do elemento 44. Na segunda paciente, constatou-se área radiolúcida em região de sínfise mentoniana, envolvendo os elementos dentários 31,32 e 41. Entretanto o elemento 41 apresentou uma área radiopaca sugestiva de calcificação. Nos casos citados, os resultados dos testes de sensibilidade pulpar estavam nos padrões de normalidade. O diagnóstico de ambos os casos foi, displasia cemento-óssea periapical. Diante deste diagnóstico, foi instituído, a proservação por 6 anos, não sendo necessárias intervenções endodôntica ou cirúrgica. Conclusão: A abordagem dos casos clínicos, ressaltou a importância da correlação entre anamnese, testes de sensibilidade pulpar e exames complementares por imagem, para o diagnóstico da displasia cemento-óssea, que comumente acometem a cavidade oral, sendo assintomáticas na maioria dos casos.
\end{abstract}

Palavras-chave: Diagnóstico; Displasia cemento óssea; Assintomática.

\section{Abstract}

Objective: To report two distinct clinical cases of periapical cemento-osseous dysplasia, with different radiographic manifestations and the same dental procedure for its resolution. Methodology: Two clinical cases were described, of female patients, black, between the third and fourth decades of life, referred to a school clinic in the Southwest of Bahia for endodontic evaluation. After the radiographic examinations, in the first patient, a radiolucent area was observed in the periapical region of element 44. In the second patient, a radiolucent area was found in the mental symphysis region, 
involving dental elements 31,32 and 41. However, element 41 presented a radiopaque area suggestive of calcification. In the cases mentioned, the results of the pulp sensitivity tests were within the normal range. The diagnosis of both cases was periapical cemento-osseous dysplasia. In view of this diagnosis, follow-up for 6 years was instituted, with no need for endodontic or surgical interventions. Conclusion: The approach to clinical cases highlighted the importance of the correlation between anamnesis, pulp sensitivity tests and complementary imaging tests for the diagnosis of cementoosseous dysplasia, which commonly affect the oral cavity, being asymptomatic in most cases.

Keywords: Diagnosis; Cementum osseous dysplasia; Asymptomatic.

\section{Resumen}

Objetivo: Informar dos casos clínicos diferenciados de displasia cemento-ósea periapical, con diferentes manifestaciones radiográficas y un mismo procedimiento dental para su resolución. Metodología: Se describieron dos casos clínicos, de pacientes del sexo femenino, de raza negra, entre la tercera y cuarta décadas de la vida, remitidas a una clínica escolar del Suroeste de Bahía para evaluación endodóntica. Tras los exámenes radiográficos, en el primer paciente se observó un área radiotransparente en la región periapical del elemento 44. En el segundo paciente se encontró un área radiotransparente en la región de la sínfisis mental, involucrando los elementos dentarios 31,32 y 41. Sin embargo, el elemento 41 presentaba una zona radiopaca sugestiva de calcificación. En los casos mencionados, los resultados de las pruebas de sensibilidad pulpar estuvieron dentro del rango normal. El diagnóstico de ambos casos fue displasia cemento-ósea periapical. Ante este diagnóstico se instituyó un seguimiento durante 6 años, sin necesidad de intervenciones endodónticas o quirúrgicas. Conclusión: El abordaje de los casos clínicos destacó la importancia de la correlación entre la anamnesis, las pruebas de sensibilidad pulpar y las pruebas de imagen complementarias para el diagnóstico de la displasia cemento-ósea, que comúnmente afecta a la cavidad oral, siendo asintomática en la mayoría de los casos.

Palabras clave: Diagnóstico; Displasia ósea de cemento; Asintomático.

\section{Introdução}

As lesões fibro-ósseas (LFOs) fazem parte de um conjunto de patologias onde ocorre a transição do osso normal, por estroma fibroso que gradativamente sofre mineralização (Nelson et al., 2019). Na atual classificação da Organização Mundial da Saúde (OMS), o fibroma cemento-ossificante, cementoblastoma benigno, e as displasias ósseas (DO), integram a terminologia LFOs, do grupo histopatológico dos Tumores Odontogênicos (Fontenele et al., 2018).

As displasias cemento-ósseas (DCO) podem ser classificadas em três subtipos: displasia cemento-óssea periapical, com maior ocorrência na região anterior da mandíbula, de forma solitária ou múltipla (Santos et al., 2021); displasia cementoóssea focal, observada na região do quadrante posterior dos maxilares (Ravikumar et al., 2020); e displasia cemento-óssea florida, que ocorre bilateralmente na mandíbula, ou em todos os quadrantes dos maxilares (Castro et al., 2017).

Na DO pode haver variação no histopatológico conforme os estágios de maturação da lesão (Fenerty et al., 2017). Pode-se observar radiograficamente que as lesões progridem de forma semelhante, se diferenciando em estágios: estágio osteolítico, misto e osteogênico (Noual et al., 2017). São observados diferentes tons de radiolucidez e radiopacidades nas massas lobulares, restritos por halos radiolúcidos (Santos et al., 2021). As lesões de origem endodôntica são similares imaginologicamente ao primeiro estágio das DO. Desse modo, os testes de sensibilidade são imprescindíveis para o estabelecimento do diagnóstico, pois os dentes envolvidos com DO possuem vitalidade pulpar (Belo et al., 2017).

Por conseguinte, há três tipos de condutas a serem seguidas diante das DCOs: acompanhamento, tratamento tradicional ou as cirurgias invasivas (Reis et al., 2020). Na ocorrência de infiltrado inflamatório relacionada a infecção local, o tratamento cirúrgico associado com a antibioticoterapia é recomendado (Kato et al., 2019). Já em casos sem sintomatologia, é apropriado a assistência clínica e radiográfica, com o intuído de se certificar que não está ocorrendo expansão da lesão (Souza et al., 2021).

Dessa forma, o presente estudo teve como objetivo relatar dois casos clínicos de displasia cemento-óssea, evidenciando suas diferenças imaginológicas. Como resolução, tiveram a mesma conduta odontológica. 


\section{Metodologia}

Trata-se de um estudo, onde foram analisados comparativamente os registros odontológicos das respectivas pacientes, em uma instituição privada do Sudoeste da Bahia. A pesquisa foi elaborada de forma descritiva e qualitativa segundo (Souza et al., 2016), da temática em foco, detalhada para destacar suas particularidades e expor a importância do seu correto diagnóstico. Para o embasamento da fundamentação teórica, utilizou-se artigos científicos disponíveis em plataformas onlines: Scielo, PubMed, BVS salud e Lilacs, tendo como descritores as palavras-chaves: "Diagnóstico", "Displasia cemento óssea", "Assintomática", empregadas de forma combinada ou única. Fez-se a utilização do operador booleano "and" nos idiomas português, inglês e espanhol.

O presente estudo foi aprovado pelo Comitê de Ética em Pesquisa da Faculdade Independente do Nordeste, sob número CAAE: 49532521.8.0000.5578.

\section{Relato de Caso}

\section{Caso 1}

Paciente do sexo feminino, melanoderma, $4^{\mathrm{a}}$ década de vida, compareceu a uma instituição privada para avaliação odontológica de rotina. $\mathrm{Na}$ anamnese, a paciente negou alergias medicamentosas, bem como patologias de base ou uso contínuo de medicamentos. Ao exame extraoral, observados, volume e simetria facial uniforme. Através do exame clínico intraoral, observou-se restauração insatisfatória na face distal do elemento 44 .

Radiograficamente, observou-se imagem radiolúcida no periápice radicular do elemento 44 (Figura 1), sugestiva de lesão periapical de origem endodôntica. Ao teste de sensibilidade pulpar (teste térmico, pelo frio), respondeu positivamente e sem comprometimento pulpar, excluindo o diagnóstico de origem endodôntica.

Figura 1. Imagem radiográfica inicial, mostrando lesão radiolúcida circunscrita no periápice do elemento dentário 44 (imagem A).

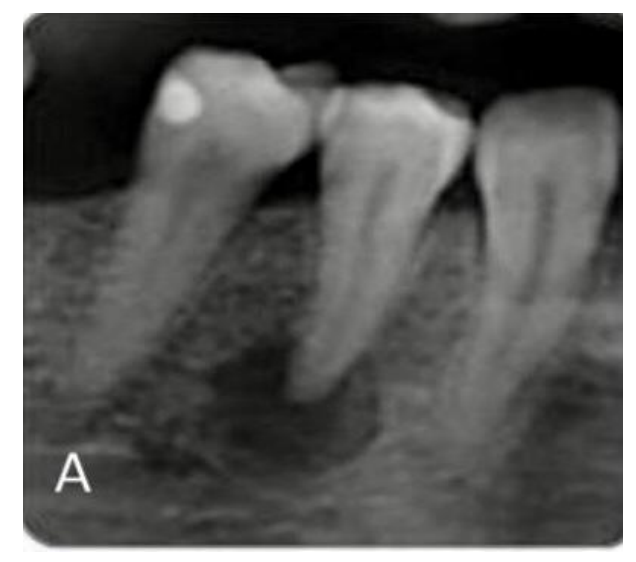

Fonte: Arquivo pessoal.

Após a realização da radiografia periapical, foi solicitado à paciente o exame panorâmico, para melhor análise e conclusão do diagnóstico. Sendo assim, foi observada a presença de uma área radiolúcida na região apical do dente 44 (figura 2). Ademais, não havia qualquer sinal de exposição óssea ou infecção na cavidade oral. 
Figura 2. Radiografia panorâmica dos maxilares.

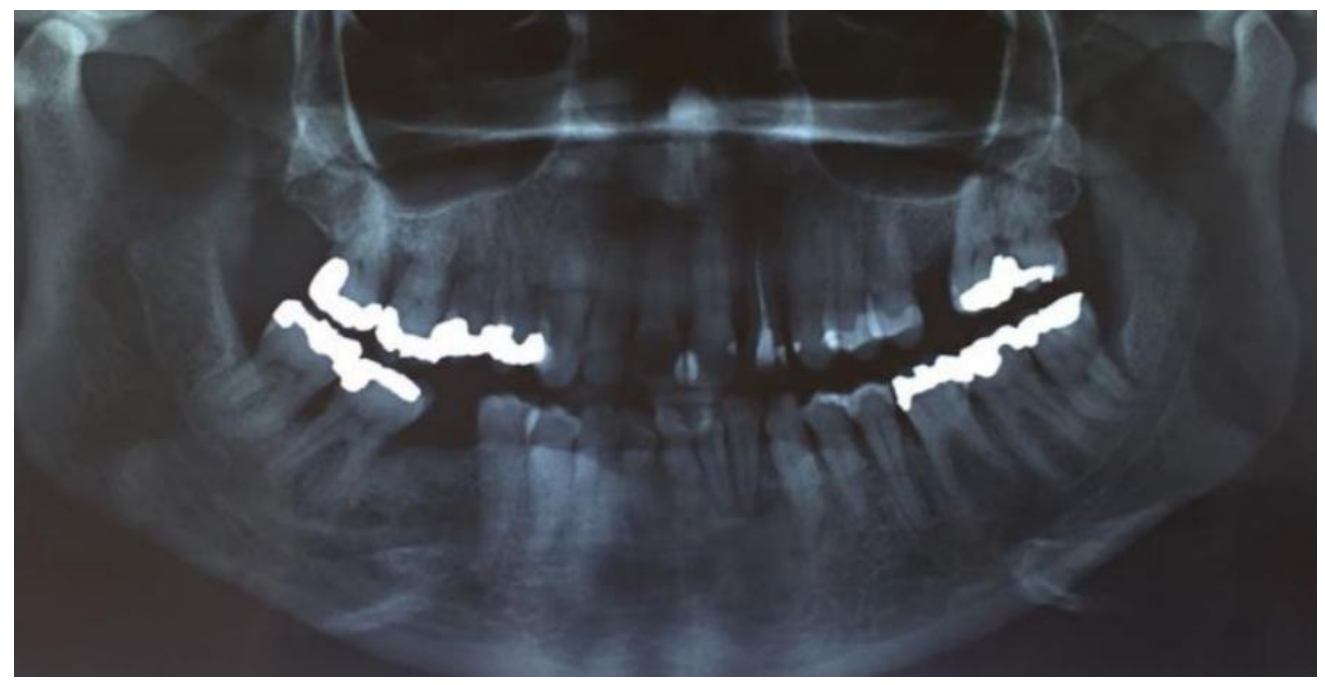

Fonte: Arquivo pessoal.

Desta maneira, associando os achados clínicos, sexo, idade, cor da paciente, achados imaginológicos, características da lesão radiolúcida (fase osteolítica), preservação da lâmina dura do dente envolvido, e a vitalidade do mesmo, foi definido o diagnóstico de Displasia cemento-óssea periapical (DCOP). Optou-se pela proservação e acompanhamento até a fase osteogênica (Figura 3) e alta da paciente.

Figura 3. Imagem radiográfica do elemento 44 após controle de 6 anos, evidenciando a calcificação da lesão (imagem B).

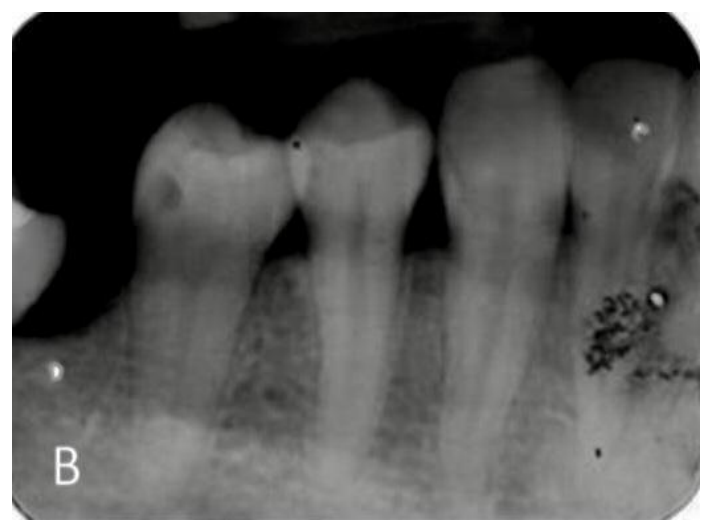

Fonte: Arquivo pessoal.

\section{Caso 2}

Paciente do sexo feminino, melanoderma, $3^{\text {a }}$ década de vida, compareceu a uma instituição privada para avaliação odontológica. No exame de anamnese, paciente negou alergias medicamentosas bem como, patologias de base, porém faz uso contínuo de medicamento anti-inflamatório não esteróide (Ibuprofeno) para tratamento de enxaquecas. Ao exame físico extrabucal, não apresentou nenhuma alteração relevante. No exame físico intrabucal, a paciente apresentava ausência do elemento dentário 46. Realizou-se radiografias periapicais da região anterior e técnica bite wing das regiões posteriores do lado direito e esquerdo, como protocolo da clínica escola.

Na região de sínfise mentoniana, observou-se área radiolúcida (Figura 4) envolvendo o periápice dos elementos dentários 31,32 e 41. No elemento 41, havia uma pequena área radiopaca, sugestiva de calcificação óssea (fase mista). 
Figura 4. Imagem radiográfica inicial da região de sínfise mentoniana (imagem C).

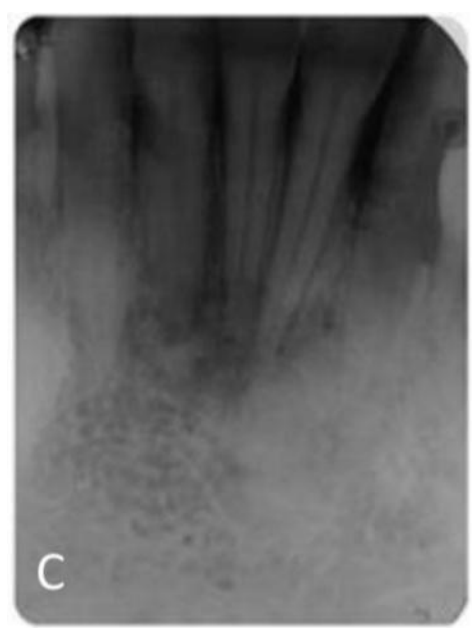

Fonte: Arquivo pessoal.

Pela possibilidade de visão global de todos os elementos, foi solicitada a radiografia panorâmica, na qual constatou imagem de densidade mista envolvendo os elementos 31,32 e 41 (Figura 5).

Figura 5. Radiografia panorâmica dos maxilares.

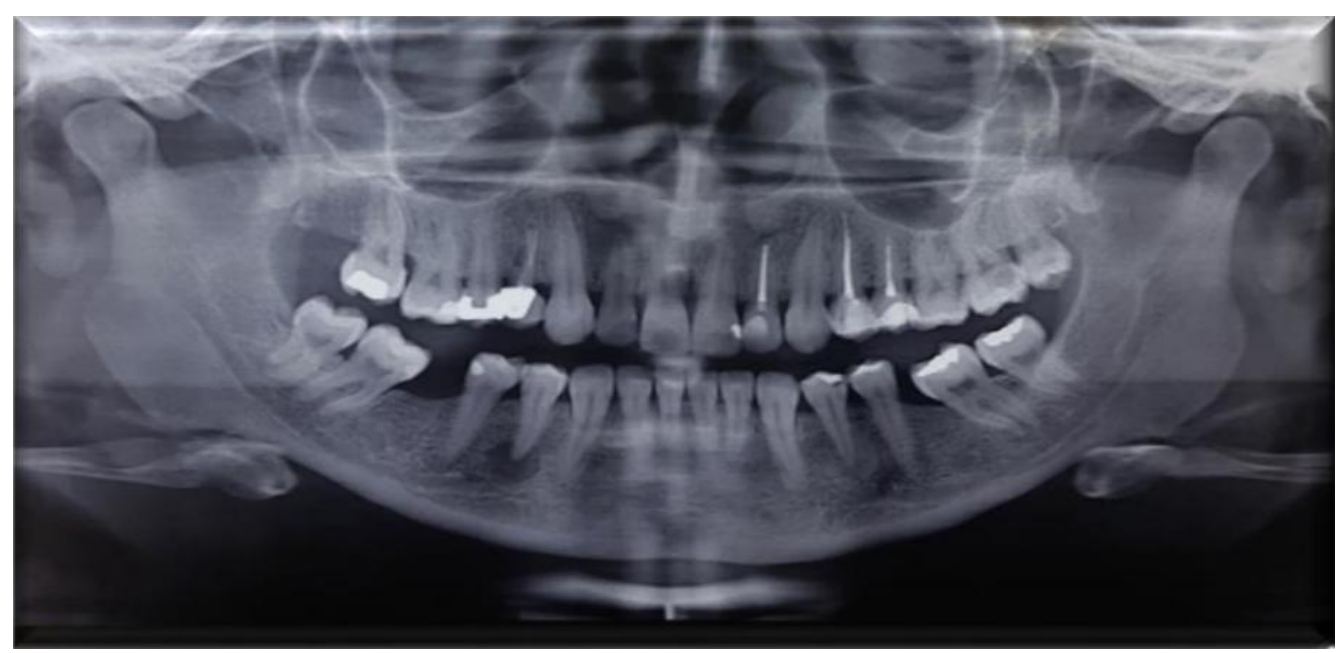

Fonte: Arquivo pessoal.

A condição da mucosa adjacente era de normalidade, e ao realizar o teste de sensibilidade pulpar (teste térmico, pelo frio) nos três elementos anteriores citados, os mesmos responderam positivamente. Diante da ausência de sinais clínicos e sintomas, e de acordo com as terminologias das LFOs, o diagnóstico foi conclusivo de Displasia cemento-óssea periapical (DCOP). Ademais, não havia qualquer sinal de exposição óssea ou infecção na cavidade oral. Sendo assim, optou-se pela proservação (Figura 7) do caso e acompanhamento até a fase osteogênica (Figura 6) e alta da paciente. 
Figura 6. Imagem radiográfica após 5 anos de controle, evidenciando completa calcificação da lesão (imagem D).

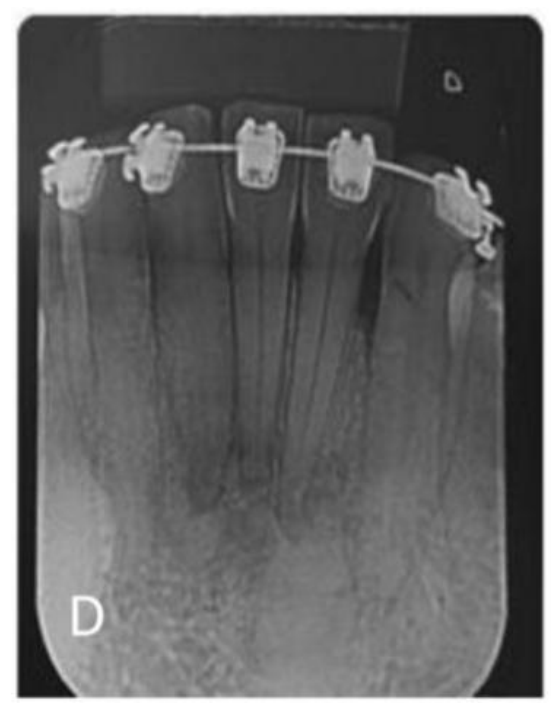

Fonte: Arquivo pessoal.

Figura 7. Imagem radiográfica após 6 anos de proservação do caso (imagem E).

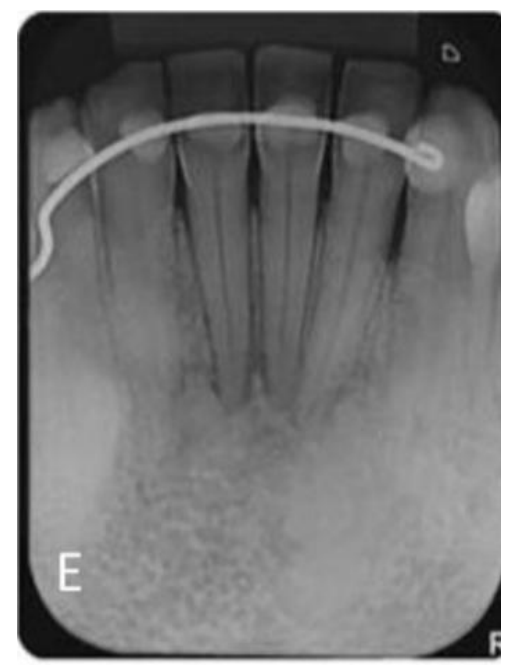

Fonte: Arquivo pessoal.

\section{Discussão}

A caracterização da DCOP, advém da modificação de tecido ósseo normal por tecido fibroso e fibras colágenas, que englobam cementóides e substâncias mineralizadas (Fontenele et al., 2018). Na microscopia, é possível verificar características semelhantes nos três subtipos, (Heitzman et al., 2019) contudo pode haver variações individuais, conforme os estágios de maturação da lesão, contemplando-se, tecido fibroso celular, osso lamenar, ausência de cápsula e tecido calcificado, dispostos em trabéculas e massas irregulares (Fenerty et al., 2017). Essas variações histológicas justificam as distintas apresentações radiográficas, como observadas nos presentes casos clínicos.

Ocasionalmente, a DCOP pode apresentar-se radiograficamente semelhante a outras patologias ósseas (Belo et al, 2017). Para diferenciar a DCOP das outras duas variantes de displasia óssea, a focal e a florida, deve-se considerar, que a primeira geralmente é observada na região posterior dos maxilares (Silva et al., 2020 (B)) limitada a região de molar, não ultrapassando $2 \mathrm{~cm}$ de diâmetro e, a segunda ocorre bilateralmente na mandíbula ou em todos os quadrantes dos maxilares (Nilius et al., 2021). 
A distinção entre a DCOP e as outras lesões do mesmo aspecto, baseia-se em dados clínicos-radiográficos, histopatológicos e demográficos.

A DCOP acomete predominantemente mulheres afrodescendentes, na faixa etária dos 30 aos 50 anos. O diagnóstico dessa patologia, geralmente, ocorre tardiamente pela escassez do serviço de saúde e/ou pela falta de conhecimento dos cirurgiões dentistas em relação as lesões fibro-ósseas, impossibilitando o diagnóstico precoce (Noual et al., 2017). No presente caso, as pacientes são do sexo feminino, entre a terceira e quarta década de vida, o que corrobora com os achados da literatura citada, sendo a predileção pela raça negra evidenciada.

As lesões são comumente encontradas em região anterior da mandíbula, podendo haver focos solitários ou múltiplos (Souza et al., 2021). Nos casos em questão, a lesão se encontrava em região posterior (caso 1), localização pouco frequente da manifestação da doença. Já no (caso 2), a lesão foi encontrada na região anterior da mandíbula.

Nos exames imaginológicos é possível observar os três estágios e suas respectivas características de acordo com seu estágio de maturação (Abramovitch \& Rice, 2015). No primeiro estágio (osteolítico), verifica-se lesão predominantemente radiolúcida/hipodensa, sendo essa, semelhante a uma rarefação óssea periapical proveniente de um processo inflamatório (Silva et al., 2020 (A)). Sua distinção é realizada através dos testes de sensibilidade pulpar, para diagnóstico diferencial das lesões de origens endodônticas (França \& Nobrega, 2020);

Lesões em estágio intermediário, imaginologicamente, apresentará aspectos de densidade mista hipodenso/ hiperdenso, notando-se, uma lesão radiolúcida com focos radiopacos no seu interior, evidenciando a substituição de tecido ósseo por tecido fibroso (Nilius et al, 2021); e por fim o estágio (osteogênico) que há predominância radiopaca/ hiperdensa, com halo radiolúcido, representando a maturação da lesão, onde ocorre a completa aposição de tecido fibroso (Nel et al., 2021). Nos casos clínicos apresentados, observamos as distintas fases imaginológicas da displasia, principalmente no caso clínico 2.

Os testes de sensibilidade pulpar são recursos suplementares ao exame clínico intraoral, sendo estes de suma importância para auxiliar no diagnóstico diferencial das lesões de origens endodônticas, uma vez que os elementos dentários envolvidos pelas displasias cemento-ósseas se mantêm com vitalidade pulpar (Castro et al., 2017). Nos casos explanados, ambos responderam positivamente ao teste térmico de frio. Radiograficamente, notou-se diferença nas manifestações das lesões. O (caso1), encontrava se no estágio osteolítico e (caso2) já estava em estágio misto.

Diferentes fatores como, idade do paciente, período de evolução em que foi descoberta, trauma na região, entre outros fatores, irão direcionar o tratamento e prognóstico (Carvalho et al., 2020). A DCOP pode predispor aos pacientes problemas como sequestros ósseos, resultando em osteomielites, pelo fornecimento reduzido de sangue em relação a densa matriz óssea acelular, conforme isso ocorre as lesões irão aumentar (Salvi et al., 2020). O acompanhamento e gerenciamento clínico dos pacientes com osteomielite secundária é mais complexo, necessitando do uso de antibióticos, mesmo que a sua eficácia seja baixa (França \& Nobrega, 2020). Manobras cirúrgicas e biópsias podem ser empregadas quando ocorrem exposições ao meio oral ou sintomatologias relevantes (Moura et al., 2018).

Em consequência aos achados clínicos e mesmo havendo diferenças imaginológicas, nos casos clínicos apresentados, nenhum tratamento foi indicado, porém ambos os casos receberam acompanhamento e proservação por 6 anos. Após calcificação significante das lesões e sem sinal de infecção na cavidade oral, as duas pacientes receberam alta, com um prognóstico favorável.

\section{Conclusão}

Constatou-se que o diagnóstico diferencial da DCOP é de suma importância e ao mesmo tempo de complexidade para os cirurgiões dentistas, pois devido às suas características radiográficas, essas lesões podem ser confundidas no seu estágio inicial com uma periapicopatia de origem endodôntica, podendo ser submetidas a um tratamento desnecessário. Para a obtenção de um diagnóstico preciso, deve ser feita a correlação entre os testes de sensibilidade pulpar, características clínicas, anamnese e 
achados imaginológicos, obtendo assim, um plano de tratamento adequado e uma conduta clinica correta, favorecendo ao paciente um prognóstico favorável.

Por consequência do presente trabalho, notamos uma escassez de estudos com acompanhamento periódico das lesões, a fim de certificar sua completa calcificação. Sugere-se, novas pesquisas de controle sobre a presente temática.

\section{Referências}

Abramovitch, K. \& Rice, D. D. (2015). Benign Fibro-Osseous Lesions of the Jaws. Dent Clin North Am., 60(1), $167-193$.

Belo, A. D. S., Silva, R. V., Pereira, R. P. \& Rodrigues, J. C. B. (2017). Importância do diagnóstico endodôntico frente à displasia cemento-óssea periapical: revisão da literatura. Dental. press endod, 7(2), 33-38.

Castro, T. F. D., Iwaki, L. C. V., Pieralisi, N. \& Silva, M. (2017). Manifestações imaginológicas distintas na displasia cemento-óssea florida. Revista da Faculdade de Odontologia, 22(2), 203-206.

Carvalho, B. O. D., Rebello, R. V., Cabral, L. N. \& Silva, M. T. B. (2020). Diagnóstico de displasia cemento-óssea florida: exames que devem auxiliar na prática clínica. Scientific Investigation In Dentistry, 25(1), 35-43.

França, K. P. D. \& Nobrega, F. (2020). Frequência de lesões compatíveis com displasia cemento-óssea em radiografias panorâmicasnde pacientes encaminhados para tratamento ortodôntico. Odontol. Clín. Cient, 19, 61-65.

Fenerty, S., Shaw, W., Verma, R., Syed, A.B., Kuklani, R., Yang, J. \& Ali, S. (2017). Florid cemento-osseous dysplasia: review of an uncommon fibro-osseous lesion of the jaw with important clinical implications. Skeletal Radiol, 46(5), 581-590.

Fontenele, R. C., Barbosa, D. A. F., Pimenta, A. V. D. M., Kurita, L. M. \& Costa, F. W. G. (2018). Importância dos aspectos imaginológicos no plano de tratamento da displasia óssea florida: Relato de caso. Rev. Cir. Traumatol. Buco-Maxilo-Fac, 18(3), 26-30.

Heitzman, L. G., Battisti, R., Rodrigues, A. F., Lestingi, J. V., Cavazzana, C. \& Queiroz, R. D. (2019). Osteomielite crônica pós-operatória nos ossos longos- O que sabemos e como conduzir esse problema*. Rev Bras Ortop., 54(6), 627-635.

Kato, C. D. N. A. D. O., Sampaio, J. D. D. A., Amaral, T. M. P. D., Abreu, L. G., Brasileiro, C. B. \& Mesquista, R. A. (2019). Oral management of a patient with cementi-osseous dysplasia: a case report. Rev Gaúch Odontol, 67, 1-8.

Moura, J. P. G., Brandão, L. B. \& Barcessat, A. R. P. (2018). Study of photodynamic (PDT) in the repair of tissue injuries: clinical case study. Estação Científica (UNIFAP), 8(1), 103-110.

Nel, C., Yakoob, Z., Schouwstra, C. M. \& Heerden, W. F. P. V. (2021). Familial florid cemento-osseous dysplasia: a report of three cases and review of the literature. Dentomaxillofac Radiol., 50(1), 1-8.

Nelson, B. L. \& Phillips, B. J. (2019). Benign Fibro-Osseous Lesions of the Head and Neck. Head and Neck Pathology, 13, 466-475.

Nilius, M., Nilius, M., Müller, C., Leonhardt, H., Haim, D., Novak, P., Franke, A., Weiland, B. \& Lauer, G. (2021). Multiple periapical dysplasia analyzed by cone-beam-computer tomografy and ${ }^{99}$ Tcm-Scintigraphy. Radiol Case, 16(12), 3757-3765

Noual, V. D., Ejeil, A. L., Gossiome, C., Moreau, N. \& Salmon, B. (2017). Differentiating early stage florid osseous dysplasia from periapical endodontic lesions: a radiological-based diagnostic algorithm. BMC oral Health, 17(1), 1-8.

Salvi, A. S., Patank, S., Desai, K. \& Wankhedkar, D. (2020). Focal cemento-osseous dysplasia: A case report with a review of literature. J oral Maxillofac Pathol., 24(1), 515-518.

Santos, A. R. D., Teixeira, N. F. D. S., Franco, A. V. D. M., Santos, V. D. C. B. D., Ferreira, S. M. S., Panjwa, C. M. B. R. G. \& Oliveira, C. R. R. D. (2021). Displasia cemento óssea florida: relato de caso. Brazilian Journal of Health Review, 4(3), 9754-9763.

Silva, A. C., Borges, K. H. D. S., Dietrich, L., Mendes, E. M. \& Sousa, G. A. (2020). Diagnóstico diferencial e conduta terapêutica para displasia óssea periapical: relato de caso. Research, Society and Development, 9(11), 1-19. (A)

Silva, D. R. D. O., Dilerato, D. P. A., Pereira, R. D. S. \& Santos, W. B. (2020). Displasia Cemento-Óssea Florida, acompanhamento clínico e radiográfico de 1 ano: relato de caso. Brazilian Journal of Health Review, 3, 563-572. (B)

Souza, L. O. D., Melo, D. D. S., Marino, B. D. C. Q. \& Soares, T. M. Z. (2016). As diferentes abordagens de pesquisa científica e suas classificações. Instituto Federal de Educação, Ciência e Tecnologia de São Paulo, 2, 1-5.

Souza, M. P. L. S., Brito, I. D. S., Oliveira, A. L. P. D. \& Marroquim, O. M. G. (2021). Displasia cemento óssea-florida tratada cirurgicamente: relato de caso. Revista Eletrônica Acervo Saúde, 13(2), 1-7.

Ravikumar, S. S., Vasupradha, G., Menaka, T. R. \& Sankar, S. P. (2020). Focal cemento-osseous dysplasia. J oral Maxillofac Pathol, 24(1), 19-22.

Reis, J. V. N. A., Nogueira, F. B. D. A., Souza, D. A. S., Santos, J. N. D. S., Ramalho, L. M. P. \& Azoubel, E. (2020). Diagnóstico precoce na displasia cementária periapical: relato de caso 7 anos de acompanhamento. Revista Portuguesa de Estomatologia, Medicina Dentária e Cirurgia Maxilofacial, 61(2), 79-85. 